\title{
Nanoporous Films with Low Refractive Index for Large-Surface Broad-Band Anti-Reflection Coatings $^{\mathrm{a}}$
}

\author{
Gaëtan Wicht, Rolando Ferrini, ${ }^{*}$ Stefan Schüttel, Libero Zuppiroli
}

Nowadays, nanoporous films are widely employed in biochemical applications or in optophotonic devices such as displays, solar cells, or light-guiding systems. In particular, the technological feasibility of nanoporous layers with low refractive indices has recently enabled the development of high-efficiency anti-reflection coatings. In this paper, we report on hybrid polymer nanoporous films that can be fabricated in a single coating step with an industrial aqueousbased method on very large surfaces. Both high transparency and low refractive index are simultaneously achieved over the entire visible spectrum. We eventually demonstrate the potential of such films for broadband AR applications by combining them in a graded-index multilayer that reduces the surface reflectivity of a polymer substrate from $10 \%$ to few $\%$.

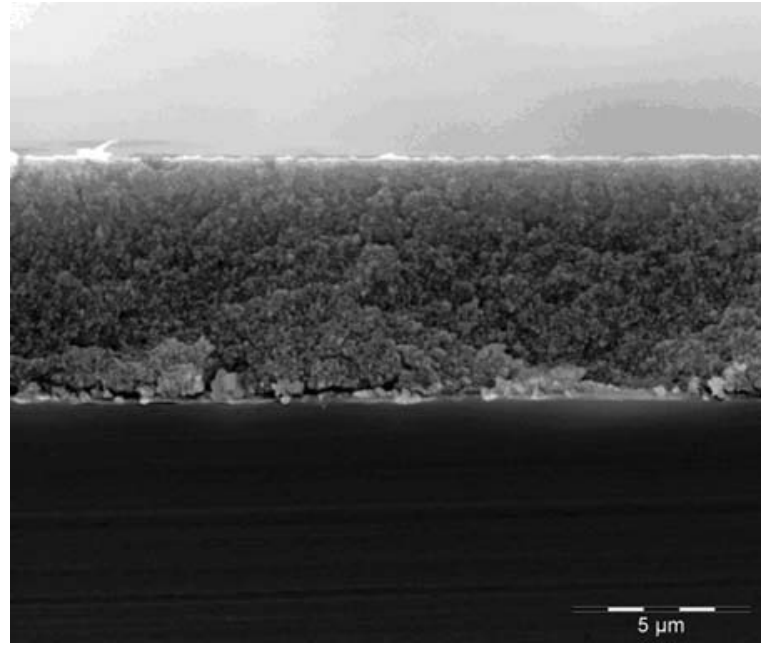

\section{Introduction}

Since the 1990s, both inorganic and organic nanoporous layers have appeared as very attractive materials with many potential applications in the fields of bio-

G. Wicht, R. Ferrini, L. Zuppiroli

Laboratoire d'Optoélectronique des Matériaux Moléculaires

(LOMM), École Polytechnique Fédérale de Lausanne (EPFL), Station 3, $\mathrm{CH}-1015$ Lausanne, Switzerland

Fax: +41 2169344 70; E-mail: rolando.ferrini@epfl.ch

S. Schüttel

Functional Materials Group, ILFORD Imaging Switzerland $\mathrm{GmbH}$, Route de l'Ancienne Papeterie, Case Postale 160, CH-1723 Marly, Switzerland

${ }^{a}$ 最 Supporting information for this article is available at the bottom of the article's abstract page, which can be accessed from the journal's homepage at http://www.mme-journal.de, or from the author. chemistry, ${ }^{[1,2]}$ electronics, ${ }^{[3]}$ optics, ${ }^{[4]}$ and photonics. ${ }^{[5]}$ In particular, their high porosity enables the fabrication of high-quality low-dielectric constant thin films that are of paramount importance for the newest micro- and optoelectronic devices. ${ }^{[6-8]}$ In the latter field, nanoporous inorganic layers, ${ }^{[9-11]}$ as well as textured polymer films, ${ }^{[12]}$ have been studied in order to improve light out-coupling from light-emitting devices. Nanostructured composite films have also been developed for optical applications such as light harvesting and energy conversion in solar cells ${ }^{[13-17]}$ or interference filters. ${ }^{[18,19]}$ The added value of nanoporous films is the possibility of achieving low refractive index $(n)$ values, which nowadays are highly demanded in several basic components of integrated optics circuits, such as, for instance, in planar waveguides for an optimized light confinement. ${ }^{[8,20,21]}$ Moreover, the introduction of low- $n$ films has represented a very important break-through in the development of interference antireflection (AR) coatings, which play a pivotal role in a wide 
variety of optical devices by increasing light transmission at the interfaces, i.e., by eliminating unwanted reflections and glare. The basic principle of AR films is known since more than two centuries: ${ }^{[22,23]}$ optical elements based on glass and common plastics have $n$ values in the range of $1.45-1.7^{[24,25]}$ and, consequently, they reflect up to $10 \%$ of normal incident light. This latter reflection can be suppressed at a given wavelength by coating a single AR layer with (i) $n_{\mathrm{f}}=\left(n_{\mathrm{s}} n_{\mathrm{a}}\right)^{1 / 2}\left(n_{\mathrm{f}}, n_{\mathrm{s}}\right.$, and $n_{\mathrm{a}}$ being the indices of film, substrate, and air, respectively) and (ii) an optical thickness that is one fourth of the reference wavelength. ${ }^{[26]}$ While condition (ii) can be easily met, condition (i) requires $n_{\mathrm{f}} \approx 1.2-1.3$ to achieve efficient AR coatings, but, unfortunately, for homogeneous dielectrics the lowest attainable $n$ values are around 1.35. ${ }^{[24,25]}$ To overcome this issue, since the 19th century, ${ }^{[23]}$ nanoporous materials have been proposed for AR applications. If the pore size is much smaller than the wavelength of light, the average $n_{\mathrm{f}}$ value can be drastically reduced with a very limited light scattering. Moreover, the possibility of tuning $n_{\mathrm{f}}$ by varying the pore size enables the fabrication of graded-index coatings. While single-layer AR coatings work only at a single wavelength and at normal incidence, these multilayer films can achieve broad-band AR performances both for polarized and unpolarized light over a wide range of incidence angles. ${ }^{[24,26]}$ Deposition or etching techniques, ${ }^{[27-31]}$ sol/gel or evaporation-induced self-assembly processes ${ }^{[32-34]}$ as well as nanoparticle coating, ${ }^{[35-39]}$ have allowed both academic and industrial researchers ${ }^{[40,41]}$ to develop inorganic nanoporous layers with $n$ values in the order of 1.1 or lower and, consequently, AR coatings with a minimum reflectivity of a few \%o in the visible spectral range. Besides these methods, nanoporous polymer coatings have lately appeared as a very attractive alternative due to their ease of processing. ${ }^{[42-50]}$ Although such organic films reach $n$ values as low as 1.1-1.2, their application is still limited by a few technological issues such as the difficulty of uniformly coating large areas and flexible polymer substrates. Finally, most of these techniques have been developed at the laboratory scale, while really costeffective industrial processes are still lacking.

In this paper, we report on hybrid polymer nanoporous films coatable in a single-step process on flexible polymer substrates over very large surfaces. An industrial roll-to-roll aqueous-based technique was used that starts from polymer suspensions of inorganic nanoparticles. Once deposited on a substrate, the solution is dried and a hybrid layer with a nanoporous morphology is formed: nanometer-size air pores appear between nanoparticle agglomerates of few hundred $\mathrm{nm}$ with the polymer acting as a simple binder. ${ }^{[11,51]}$ By properly controlling the nanoparticle surface, the solution composition or the fabrication process and, in particular, by varying the nanoparticle material, e.g., silica $\left(\mathrm{SiO}_{2}\right)$, aluminum oxide/hydroxide $(\mathrm{AlOOH})$, or titania $\left(\mathrm{TiO}_{2}\right)$, nanoporous layers are obtained with an average refractive index that can range from very low $(n<1.2)$ to low $(1.2<n<2.0)$ values in the visible spectral region. ${ }^{[11,51,52]}$ Here, we focus on highly transparent (transmission higher than $90 \%)$ very low- and low- $n(1.13<n<1.4) \mathrm{SiO}_{2}$-based films that were combined to coat graded- $n$ multilayers over polymer substrates with a precise control over the layer thickness and the optical properties. Broad-band AR films were thus obtained that reduce the surface reflection from $10 \%$ to few $\%$ in the visible region.

Since several decades, roll-to-roll techniques have enabled the coating of high quality optical and photographic films. In particular, the aqueous-based curtain and cascade coating techniques are already used to produce hybrid multilayers with a total thickness of few $\mu \mathrm{m}$ by simultaneously depositing up to eight functionalized polymer layers. ${ }^{[52]}$ In the 1990s, with the introduction of the ink-jet printing, these techniques were further developed to fabricate novel nanoporous films as printing media for photo-quality inkjet prints. Here we demonstrate that such films possess very good optical properties that can eventually be exploited for the development of opto-photonics devices such as AR coatings. With respect to other nanoporous polymer films, ${ }^{[42-50]}$ they are already produced with a versatile industrial technique that easily allows the coating of uniform and well-controlled films over large areas.

\section{Experimental Part \\ Materials}

All nanoporous layers were coated on transparent substrates consisting of a thick ( $\approx 175 \mu \mathrm{m}$ ) poly(ethylene terephthalate) (PET) Cronar $^{\mathbb{R}} 742$ film covered by a 100-nm adhesion layer on both faces (DuPont Teijin Films). These latter adhesion layers consist in a nonporous coating from a mixture of different polymers that enables the adhesion of hydrophilic layers obtained from water-based coating procedures on the PET substrate. Polyvinyl alcohol (PVA) and boric acid (or sodium tetraborate) were used as binder polymer and hardener, respectively. The coating solution was prepared using: (i) $\mathrm{SiO}_{2}$ nanoparticles CAB-O-SIL/H5 and M5 with a primary particle size of $8 \mathrm{~nm}$ ["small nanoparticles" (SNPs)] and $12 \mathrm{~nm}$ ["large nanoparticles" (LNPs)], respectively (Cabot GmbH); (ii) AlOOH nanoparticles Disperal AP/3 and HP14/4 with a primary particle size of 8 and $14 \mathrm{~nm}$, respectively (SASOL Germany $\mathrm{GmbH}$ ); (iii) $\mathrm{TiO}_{2}$ nanoparticles VP Disp W 740 X directly dispersed in water with particulate distributions well below $100 \mathrm{~nm}$ aggregate sizes (Degussa).

\section{Film Coating}

The $\mathrm{SiO}_{2}$-based films were fabricated using a Meyer-bar coater and different coating solutions were prepared by dispersing SNPs and LNPs in a PVA solution. ${ }^{[11-51]}$ We highlight the fact that the film porosity (i.e., its refractive index) is strongly affected by both the 
PVA content and the intended film thickness. Therefore, the composition of such solutions was set to minimize the PVA content (i.e., to maximize the film porosity) while keeping an acceptable mechanical stability. Two different sets of samples were obtained depending on the size of the primary $\mathrm{SiO}_{2}$ nanoparticles, i.e., SNP and LNP films. For these latter layers the surface of the $\mathrm{SiO}_{2}$ nanoparticles was modified by applying the method described in ref., ${ }^{[51]}$ while for the SNP films the $\mathrm{SiO}_{2}$ nanoparticles were used as received. Typical LNP-coating solutions were prepared by dispersing the nanoparticle powder in deionized water at $40^{\circ} \mathrm{C}$ with a Branson sonifier, while for the SNP films the $\mathrm{SiO}_{2}$ nanoparticles were dispersed with a high-sheer mixer at room temperature at a pH above 8.5 (i.e., $5-10 \mathrm{mg} \mathrm{NaOH}$ per $\mathrm{g} \mathrm{SiO}_{2}$ ). The PVA binder was prepared as a separate solution and combined with a surfactant (10G from Olin Corp.), the nanoparticle dispersion and, shortly before the film deposition, the hardener (boric acid or sodium tetraborate). Typical compositions of the coating solution for the SNP and LNP films with a ratio $\mathrm{SiO}_{2} / \mathrm{PVA}=7: 1$ and 6:1, respectively, are the following: (i) $36 \mathrm{~mL}$ water, $2.5 \mathrm{~g} \mathrm{SiO}_{2}$ nanoparticles, $0.35 \mathrm{~g}$ PVA, and $0.02 \mathrm{~g}$ sodium tetraborate; (ii) $42 \mathrm{~mL}$ water, $5.4 \mathrm{~g} \mathrm{SiO}_{2}$ nanoparticles, $0.9 \mathrm{~g} \mathrm{PVA}$, and $0.2 \mathrm{~g}$ boric acid. After coating the solution, the SNP and the LNP layers were dried in an oven for 30 min with a laminar flow of dry air at $45^{\circ}$.

Similar coating procedures were adopted to fabricate the $\mathrm{AlOOH}$ - and $\mathrm{TiO}_{2}$-based films. Concerning the preparation of the coating solutions, while the $\mathrm{AlOOH}$ primary nanoparticles were used as received and acids were added to favor their dispersion, the $\mathrm{TiO}_{2}$ nanoparticles were directly purchased as dispersions.

For the AR multilayers, the coating solutions were prepared using the $\mathrm{SiO}_{2}$-SNPs. Two nanoporous layers with low and high porosity (i.e., high and low $n$, respectively) were then coated one on top of the other. Standard compositions of the coating solutions for the low porosity/high- $n$ and the high porosity/low- $n$ layers with a ratio $\mathrm{SiO}_{2} / \mathrm{PVA}=0.9: 1$ and 4:1, respectively, are the following: (i) $35 \mathrm{~mL}$ water, $0.8 \mathrm{~g} \mathrm{SiO}_{2}$ nanoparticles, $0.9 \mathrm{~g}$ PVA, and $0.01 \mathrm{~g}$ sodium tetraborate; (ii) $35 \mathrm{~mL}$ water, $1.3 \mathrm{~g} \mathrm{SiO}_{2}$ nanoparticles, $0.3 \mathrm{~g}$ PVA, and $0.01 \mathrm{~g}$ sodium tetraborate. After coating the solution, the layers were dried in an oven with a laminar flow of dry air at $45^{\circ} \mathrm{C}$ for $30 \mathrm{~min}$. We observe that, since the adhesion layer is a polymerbased layer specifically designed to adhere to the PET substrate, as well as to any hydrophilic layer obtained by water-based coating procedures, the adhesion of the first nanoporous monolayer is always excellent. All the additional layers coated on top of the first layer have a very similar composition and will thus adhere perfectly to it. Moreover, as it will be discussed in the following, our nanoporous layers have an intrinsic surface roughness that depends mainly on the size of the nanoparticle agglomerates, the dispersion quality and, on a larger scale, the coating process. Due to the high dilution of the coating solution, a second layer coated on top of a slightly rough dried layer will partly level out the induced roughness without replicating it but increasing the roughness of the top surface. That is, the interface roughness between two nanoporous layers and the top surface roughness are always very similar.

All the layer thicknesses were generally measured as explained below and compared to the intended values. If necessary, this latter result was used to fine-tune the coating thickness by adapting the $\mathrm{SiO}_{2}$ and the PVA concentrations of the coating solutions.

\section{Film Characterization}

Scanning electron microscopy (SEM) micrographs were taken with a JEOL JSM 6400 at the highest possible resolution $($ magnification $=5000 \times)$. The samples were prepared with a Leica microtome. The cut procedure was optimized to obtain cleaved edges suitable to determine the layer thickness and to analyze the particle agglomeration. A thin $(\approx 30 \mathrm{~nm})$ gold layer was deposited on the surface of the nanoporous films as conducting layer. The mean film thicknesses were obtained by averaging several measurements taken at different positions on the sample surface.

Atomic force microscopy (AFM) measurements were performed with a scanning probe microscope NT-MDT Solver PRO-M in semicontact mode with $\mathrm{Si}$ cantilevers. Tips were chosen with a curvature radius of $10 \mathrm{~nm}$. The surface homogeneity was checked by repeating measurements at several positions on the film surface over $5 \times 5 \mu \mathrm{m}^{2}$ regions.

\section{Optical Measurements}

Standard specular reflectance and transmittance measurements were performed by means of an optical bench equipped with an integrating sphere (Oriel, mod. 70679), a standard halogen lamp as illumination source $(12 \mathrm{~V}, 100 \mathrm{~W})$ and a high-resolution spectrophotometer composed by a monochromator (Jobin-Yvon, mod. HR460) coupled to a Si CCD detector. Both the total and the specular reflectance/transmittance were measured in the visible spectral range.

The optical characterization was completed using a spectroscopic ellipsometer with a rotating polarizer (Sopra, mod. GESP5). For the ellipsometric measurements, the uncoated surface of the film substrate was roughened and covered with a black absorbing paint in order to suppress the light reflection from the lower sample surface. The standard procedure described in ref. ${ }^{[53]}$ was used to calculate the polarization degree.

The refractive index of the bare $\mathrm{AlOOH}$ nanoparticles was roughly estimated using refractive index matching liquids and found to be close to that of $\mathrm{Al}_{2} \mathrm{O}_{3}$, i.e., in the order of 1.645 at $550 \mathrm{~nm}$.

Concerning the optical characterization of the AR multilayers, the PET substrates were coated either on one or two surfaces for ellipsometric and reflectance measurements, respectively. In the first case, the uncoated surface was treated as for the nanoporous monolayers in order to suppress the light reflection at the bottom surface.

Specular reflectance measurements as a function of both polarization and angle of incidence were performed using the ellipsometer in the photometry mode, i.e., as a standard polarization-sensitive spectrophotometer with angular resolution.

\section{Results and Discussion}

\section{Nanoporous Monolayers}

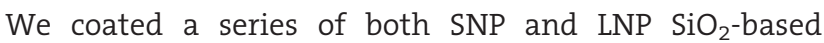
monolayers on transparent PET substrates. The precursor solutions were deposited on one side of the substrate covered by a 100-nm adhesion layer (Figure 1). It is during 
b)

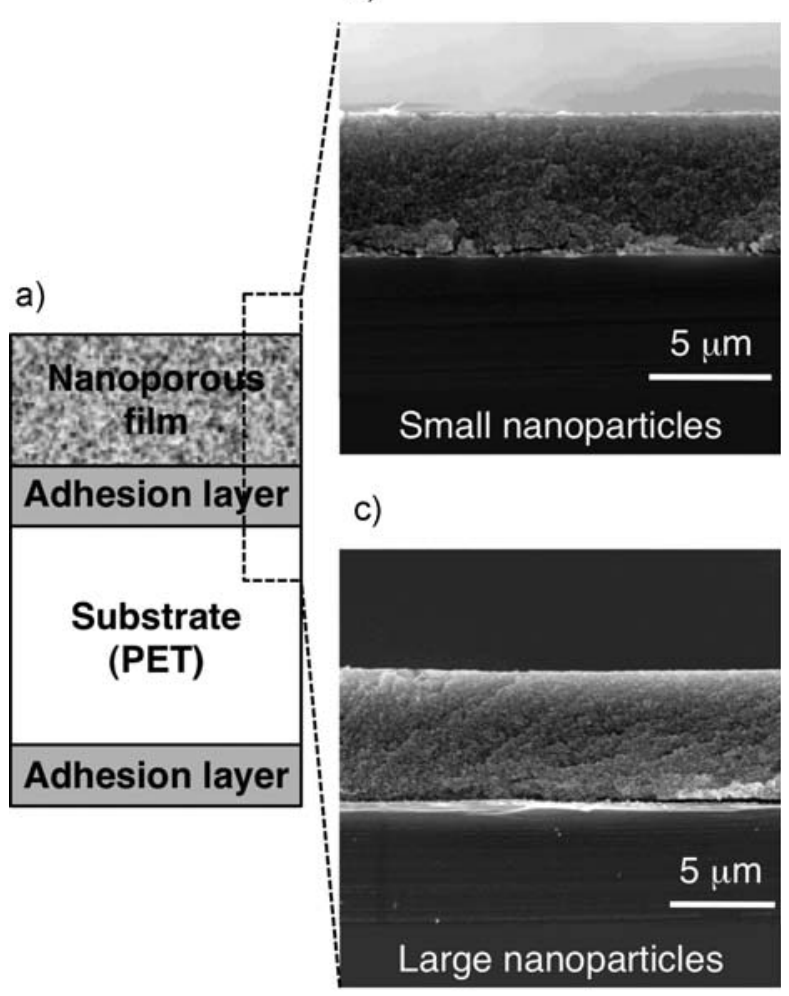

d)

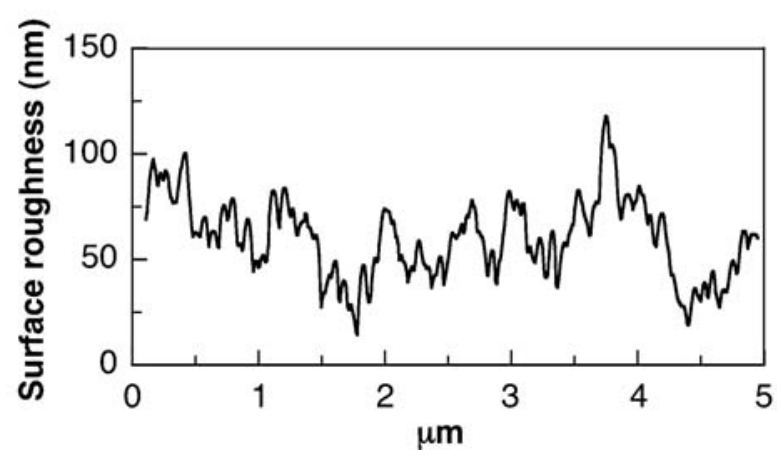

Figure 1. (a) Sketch of the vertical structure of a hybrid polymer nanoporous film coated on a PET substrate covered by a 100-nm adhesion layer on both faces. (b) and (c) SEM images of $\mathrm{SiO}_{2}$ based nanoporous films coated from "small" [8 nm (SNP)] and "large" [12 nm (LNP)] primary nanoparticles, respectively. (d) A typical surface roughness profile of a $\mathrm{SiO}_{2}$-based nanoporous film as measured by AFM.

the drying phase after the film coating that our hybrid polymer layers develop their typical porous morphology: the primary nanoparticles form larger agglomerates in the order of 10 to some $100 \mathrm{~nm}$ with the PVA acting as a simple binder, while air pores appear in the layer bulk. The resulting porosity as well as the pore size are the critical factors that eventually determine the optical properties of such films. ${ }^{[46]}$ On one hand, increasing the porosity decreases the average $n$ value of the layer. On the other hand, if the pore size is comparable to or larger than the visible wavelengths, the film appears to be opaque due to the light scattering. On the contrary, if the length scales of the porous structure are much lower than the visible wavelengths (i.e., smaller than a few hundred $\mathrm{nm}$ ), the light scattering becomes negligible and the nanoporous films appear to be transparent. ${ }^{[42,54]}$

Both the layer and the surface morphologies were investigated by SEM and AFM. The SEM images of both the SNP and the LNP films are shown in Figure $1(b)$ and (c), respectively. The film thickness ( 5 and $6 \mu \mathrm{m}$ for the SNP and the LNP films, respectively) was roughly estimated by averaging several measurements taken at different positions on the sample surface. The SEM micrographs reveal a homogeneously porous morphology with pore sizes in the order of several $10 \mathrm{~nm}$, i.e., below the $100-\mathrm{nm}$ threshold where the visible light starts to be scattered.$^{[42,54]}$ Moreover, because of the very low $n$-contrast between the $\mathrm{SiO}_{2}$ and the film matrix, the light scattering from the nanoparticle agglomerates dispersed in the layer bulk is very moderate and our nanoporous layers are indeed highly transparent: transmittance $(T)$ and specular reflectance $(R)$ measurements over the entire visible spectrum are reported in Figure 2 for both the SNP and the LNP films. The interference
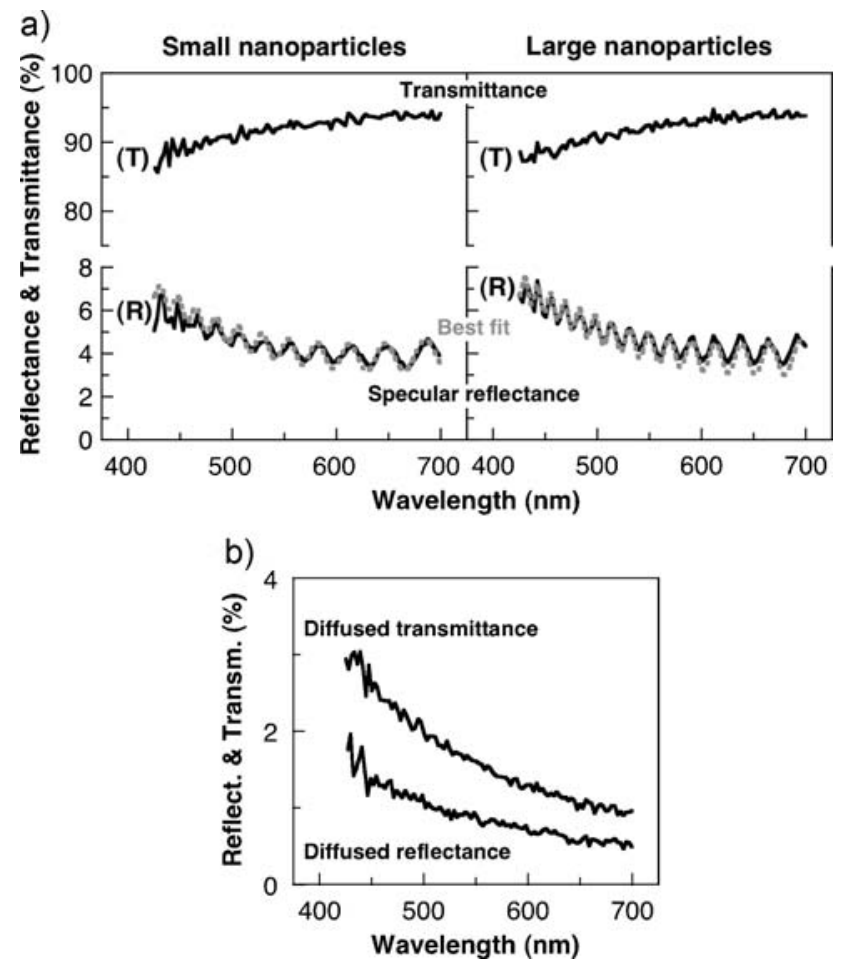

Figure 2. (a) Specular reflectance $(R)$ and transmittance $(T)$ spectra of the $\mathrm{SiO}_{2}$-based nanoporous films shown in Figure 1. The best fit of the specular reflectance spectra obtained using the ellipsometric data reported in Figure 3 are shown (gray dotted lines). (b) Diffused reflectance/transmittance spectra of a typical $\mathrm{SiO}_{2}-$ based nanoporous film. 
fringes that appear in both $T$ and $R$ spectra are due to the multiple interferences at the film-substrate and the film-air interfaces. We highlight that for all our nanoporous films the average $R$ is as low as $5 \pm 1 \%$, while $T$ reaches values well above 90\%. Nevertheless, the AFM measurements show a slight surface roughness (some tens to hundreds $\mathrm{nm}$ ) due to the presence of nanoparticle agglomerates at the film surface [Figure $1(\mathrm{~d})$ : note that, since AFM images are always a convolution of the surface topography with the tip shape, the $10-\mathrm{nm}$ curvature radius of our tips limits the resolution for the smallest features]. Since these agglomerates are almost uncapped, they can actually originate a moderate light scattering: as it is shown in Figure 2(b), residual diffused transmittance and reflectance contributions in the order of $1-3 \%$ were indeed measured for all the studied samples.

Ellipsometric measurements were performed on all samples (Figure 3). A polarization degree larger than 95\% was measured over the entire visible spectrum, ${ }^{[53]}$ thus confirming that (i) in spite of their nanoporous morphology, our hybrid films can be considered as homogeneous with regard to their macroscopic optical properties, and (ii) no depolarization effects are introduced by the residual surface

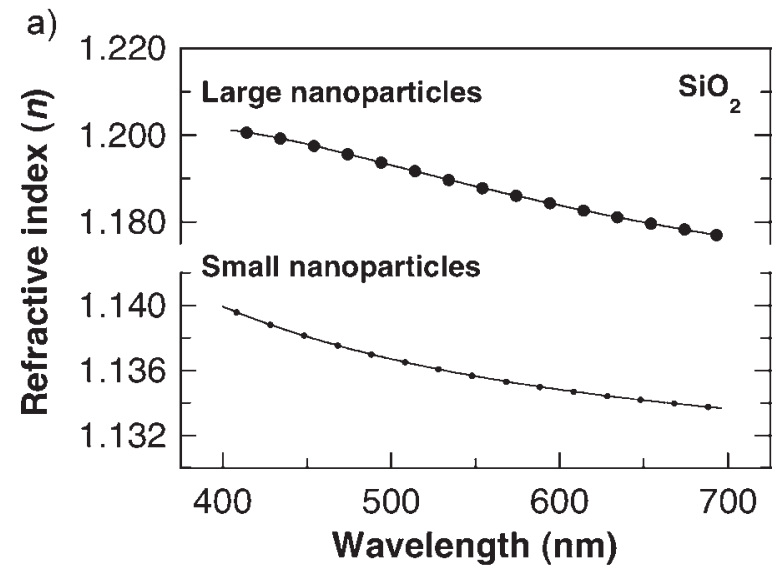

b)

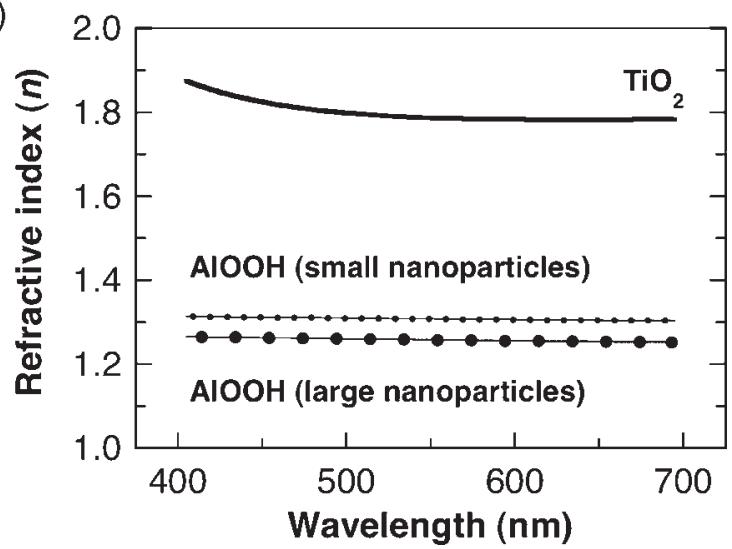

Figure 3. Refractive index spectra of (a) the $\mathrm{SiO}_{2}$-based nanoporous films shown in Figure 1, and (b) the $\mathrm{AlOOH}$ - (small and large primary nanoparticles) and the $\mathrm{TiO}_{2}$-based nanoporous layers. roughness [Figure $1(d)$ ] and the consequent moderate light scattering [Figure 2(b)]. Therefore, in order to fit the ellipsometric spectra, we adopted a standard multi-layer model that includes an effective layer mimicking the contribution of the surface roughness [Figure $1(\mathrm{~d})]^{[55]}$ The thickness $(105 \pm 10 \mathrm{~nm})$ and the refractive index of the adhesion layer and of the PET substrate were obtained by separate ellipsometric measurements (Figure 4). The free fitting-parameters were the thickness $t_{\mathrm{npl}}$ and the refractive index $n$ of the nanoporous film as well as the thickness $t_{\text {rou }}$ and the air percentage of the roughness layer (see the Supporting Information for a description of the fitting

a)
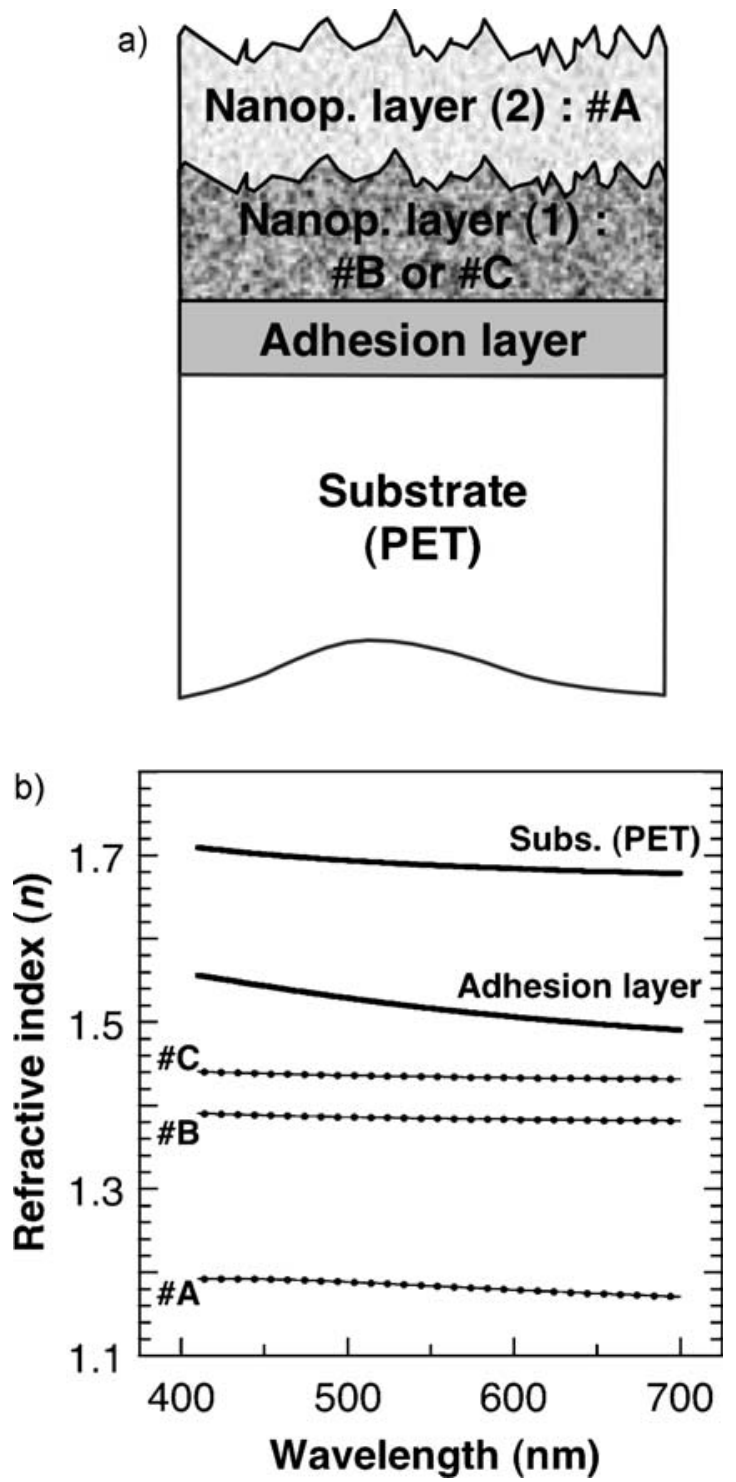

Figure 4. (a) Sketch of the fabricated AR coatings. (b) Refractive index spectra obtained from the ellipsometric measurements of single A, B, and C nanoporous layers (black thin lines and dots). The $n$ values for the PET substrate and the adhesion layer are also shown (black thick lines). 
model). A 50\% air content was found in these latter layers for all samples, with the fitted $t_{\text {rou }}$ and $t_{\text {npl }}$ values in good agreement with the SEM and the AFM measurements: i.e., $\quad t_{\text {rou }}=120$ and $150 \pm 5 \mathrm{~nm}$, and $t_{\mathrm{npl}}=4.81$ and $6.19 \pm 0.01 \mu \mathrm{m}$ for the SNP and the LNP films, respectively. The $n$ values stemming from the fit of the ellipsometric spectra are reported in Figure $3(\mathrm{a})$ : they yield an average layer porosity in the order of $47 \%$ and $56 \pm 1 \%$ for the SNP and LNP films, respectively. ${ }^{[56]}$ In order to check the reliability and the precision of the obtained $n$ values, they were used to fit the corresponding $R$ spectra [Figure 2(a): gray dotted lines]. To this aim, a transfer-matrix method was adopted that models the nanoporous films as a homogeneous layer with an average thickness $\left\langle t_{\text {npl }}\right\rangle$ and a roughness root mean square $\Delta_{\text {rou }}$ and takes into account the surface/interface light scattering basing on the model of ref. ${ }^{[57,58]}$ (see the Supporting Information for a description of the fitting model). The fitted $\left\langle t_{\text {npl }}\right\rangle$ and $\Delta_{\text {rou }}$ values were in very good agreement with the ellipsometric results: i.e., $\left\langle t_{\mathrm{npl}}\right\rangle=5.95$ and $6.48 \pm 0.01 \mu \mathrm{m}$ with $\Delta_{\text {rou }}=80$ and $60 \pm 5 \mathrm{~nm}$ for the SNP and the LNP layers, respectively.

We highlight that both SNP and LNP films are characterized by $n$ values lower than 1.2 over the whole visible spectrum with negligible refractive index dispersion. In particular, in the SNP layers we could achieve very low $n$ values in the order of 1.13-1.14 with high transparency $(T>90 \%)$ and very moderate residual light scattering $(\approx 1-$ $3 \%$ ). The advantage of our coating technique with respect to other procedures is its intrinsic versatility. On one hand, the choice of the nanoparticle size (e.g., "small" vs. "large" nanoparticles) and their eventual surface treatment, as well as the formulation of the coating solution (e.g., the polymer content), have an important impact on the achievable degree of porosity, thus enabling the fine-tuning of the intended $n$ values. On the other hand, the possibility of choosing among several nanoparticle materials (e.g., $\mathrm{SiO}_{2}, \mathrm{AlOOH}$, and $\mathrm{TiO}_{2}$ ) gives access to different refractive index windows from very low $(n<1.2)$ up to low $(1.2<n<2.0)$ values. Namely, the refractive index of the oxide nanoparticles controls the range of $n$ that can be achieved, with the $\mathrm{SiO}_{2}$ - and $\mathrm{TiO}_{2}$-based films covering the very low and the high end of the scale, respectively. Therefore, in order to thoroughly demonstrate the potential of our method, we fabricated $\mathrm{AlOOH}$ - (with small and large primary nanoparticles) and $\mathrm{TiO}_{2}$-based nanoporous monolayers with morphological properties similar to those of the $\mathrm{SiO}_{2}$-based films described above: their refractive index spectra are shown in Figure 3(b). Finally, we observe that such nanoporous films are state-ofthe-art components in the imaging industry and serve as absorbing layers for ink-jet printing materials. ${ }^{[52]}$ These films yield excellent performances in adverse light and climate conditions and have reasonable chemical stability (e.g., excellent water stability). Layer adhesion and brittleness are well understood as the layers are typically applied to flexible substrates. However, due to their high porosity, mechanical durability is limited and staining by absorption of dirt or colored liquids is possible.

\section{Graded-Index Nanoporous AR Multilayers}

With the possibility of coating in a single fabrication step hybrid polymer multilayers consisting of several nanoporous layers, while adjusting independently the layer thicknesses and their porosity (i.e., their optical properties), our hybrid polymer nanoporous films are suitable to develop complex optical devices like, for instance, AR coatings. Therefore, we designed and fabricated a gradedindex three-layer structure consisting of two $\mathrm{SiO}_{2}$-based nanoporous layers with low (lower layer) and high (upper layer) porosity (i.e., high and low $n$, respectively) on top of the adhesion layer [Figure 4(a)]. The same coating procedure used for the SNP films was adopted and, being the PET film transparent, the same AR coating was fabricated on both sides of the plastic substrate. The compositions of the coating solutions were chosen to adjust both the thicknesses and the refractive index of the nanoporous layers so to minimize the reflectivity of the coated substrate at certain wavelengths in the $500-600 \mathrm{~nm}$ spectral interval. ${ }^{[26]}$ Two AR films were fabricated changing the lower layer (B or C) while keeping the same upper layer (A) [layers (1) and (2) in Figure 4(a), respectively]. Figure 4(b) presents the $n$ spectra of the basic building blocks as measured independently by ellipsometry. The $R$ and $T$ spectra of the PET substrate both uncoated (black lines) and coated on both surfaces with the $\mathrm{AB}$ (red lines) and the $\mathrm{AC}$ (blue lines) films are shown in Figure 5. Note that the presence of either the $\mathrm{AB}$ or the $\mathrm{AC}$ multilayer reduces the film reflectance to values in the order of few $0.1 \%$ s over the entire visible spectrum, while the transmittance increases correspondingly from $85 \%$ (PET) to values higher than 95\%. Ellipsometric and reflectance spectra of both AR films were fitted using an extension of the models adopted for the monolayer samples (see the Supporting Information for a description of the fitting models). From the best fit of the ellipsometric spectra we obtained $t_{\mathrm{npl}}(1) /(2)=122 / 365$ and $326 /$ $820 \pm 10 \mathrm{~nm}$ with $t_{\text {rou }}(1) /(2)=117 / 69$ and $117 / 89 \pm 5 \mathrm{~nm}$ for the $A B$ and the AC films, respectively, in agreement with the fit of the reflectance spectra [dotted lines in Figure $5(\mathrm{a})]^{[57]}$ yielding $\left\langle t_{\mathrm{npl}}\right\rangle(1) /(2)=260 / 450$ and $450 /$ $860 \pm 10 \mathrm{~nm}$ with $\Delta_{\text {rou }}(1) /(2)=60 / 45$ and $60 / 50 \pm 5 \mathrm{~nm}$ for the $A B$ and the $A C$ films, respectively.

We observe that, while the intensity of the residual light scattering from the film surface is very moderate $(\leq 1-3 \%)$, the interference properties of our nanoporous multilayers are influenced by the interface roughness. Due to the presence of a roughness root mean square in the order of some ten nm, the interference maxima and minima in the $R$ spectra are smoothed and broad spectral regions are obtained where the 


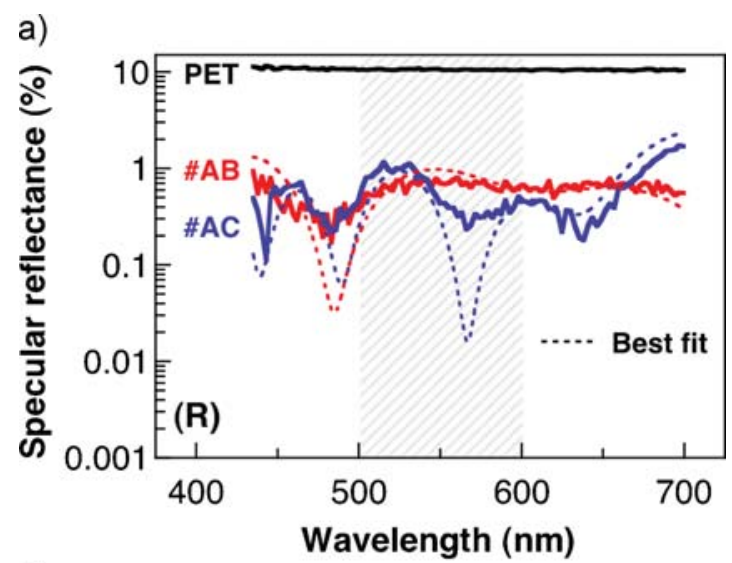

b)

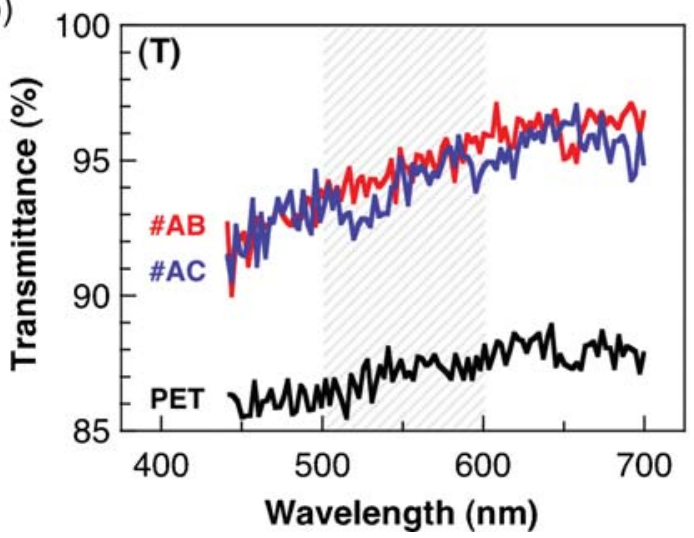

Figure 5. (a) Reflectance and (b) transmittance measurements on the PET substrate coated on both surfaces with the $A B$ and the $A C$ films sketched in Figure 4(a) (red and blue lines, respectively). The spectra of the uncoated PET substrate are reported as reference (black line). The best fits of the specular reflectance spectra obtained using the ellipsometric data reported in Figure $4(b)$ are shown (dotted lines). The gray background indicates the spectral region where the reflectance is intended to be minimized according to the model of ref. ${ }^{[26]}$

reflectivity is almost constant with a measured experimental value as low as $0.1 \%$. Note that, as it is shown by the fit in Figure 5(a) (dotted line), theoretical values in the order of few $0.01 \%$ s may be actually achieved for few given wavelengths. The design of multi-layer films with an optimized gradedindex profile ${ }^{[28]}$ would certainly allow us to lower the experimental $R$ value while keeping the typical broad-band response that is one of the main assets of our nanoporous AR coatings together with their large surface.

A second set of the AC films was fabricated with different layer thicknesses: the values obtained from the fit of the reflectance spectra are $\left\langle t_{\mathrm{npl}}\right\rangle(1) /(2)=300 / 650 \pm 10 \mathrm{~nm}$ $\left[\Delta_{\text {rou }}(1) /(2)=70 / 55 \pm 5 \mathrm{~nm}\right]$, while from the ellipsometric measurements $t_{\mathrm{npl}}(1) /(2)=200 / 548 \pm 10 \mathrm{~nm}\left[t_{\text {rou }}(1) /\right.$ $(2)=130 / 90 \pm 5 \mathrm{~nm}$. The AR properties of this $\mathrm{AC}(2)$ coating are clearly shown in Figure 6, where the optical photographs of a printed text [Figure 6(a)] and a light source [Figure $6(b)]$ that reflect on both an uncoated and a coated
PET surface are presented. With our hybrid polymer AR multilayers on both sides, the transparency of the PET film is clearly increased and, correspondingly, the residual glare is reduced, i.e., the reflected image of both the text and the source is completely suppressed. The $R$ spectra of both the uncoated and the coated PET substrate are reported in Figure 6 (c). We observe that the measured reflectivity of the optimized $\mathrm{AC}(2)$ coating is almost constant (in the order of few $0.1 \%$ s) over the entire visible spectrum and its average value is slightly lower than for the previous AC film, while the minimum of the simulated $R$ spectrum (dotted line) does reach values down to several $0.001 \%$.

Finally, in order to thoroughly characterize the AR properties of our graded-index nanoporous multilayers, ${ }^{[24]}$ we measured both the unpolarized and the polarized (with both $s$ - and $p$-polarized light) $R$ spectra of the $\mathrm{AC}(2)$ film at variable incidence angle from $0^{\circ}$ (normal incidence) up to $60^{\circ}$ and we compared them with the corresponding spectra of the uncoated PET substrate. The experimental spectra measured at 0 and $40^{\circ}$ are reported in Figure 7 (gray and black lines, respectively). With unpolarized light the AR performances of
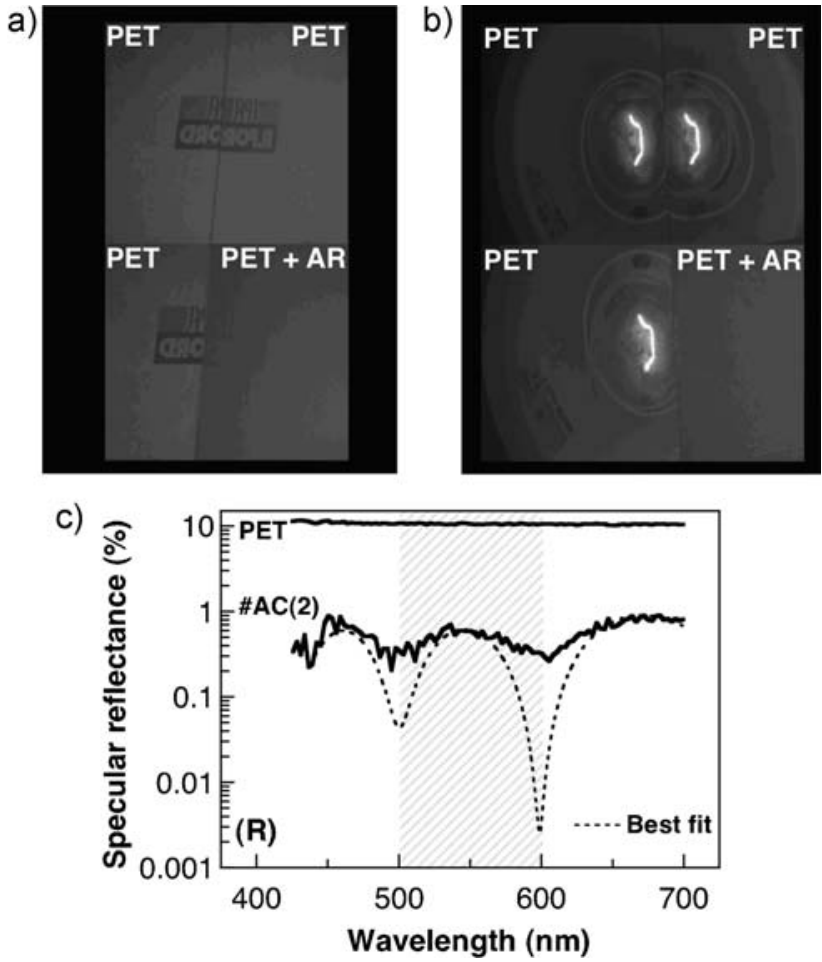

Figure 6. (a) and (b) Photographic images of a printed text and a light source, respectively, that reflect on an a PET substrate either uncoated or coated with an optimized graded-index nanoporous multilayer [namely, an $\mathrm{AC}(2)$ film with optimized layer thicknesses]. (c) Specular reflectance spectrum in presence of the AR film compared with the spectrum of the uncoated PET substrate. The best fit of the reflectance spectrum obtained using the $n$ data reported in Figure $4(b)$ is shown (dotted lines). The gray background indicates the spectral region where the reflectance is intended to be minimized according to the model of ref. ${ }^{[26]}$ 
our hybrid polymer coatings are not affected by variations of the incidence angle for values up to $50^{\circ}$ : the reflectivity of the coated substrate remains in general 1-2 orders of magnitude lower than that of the bare PET [see Figure 7(a)]. Moreover, for the same incidence angles and in presence of polarized light, the AR response is almost polarization-insensitive [see
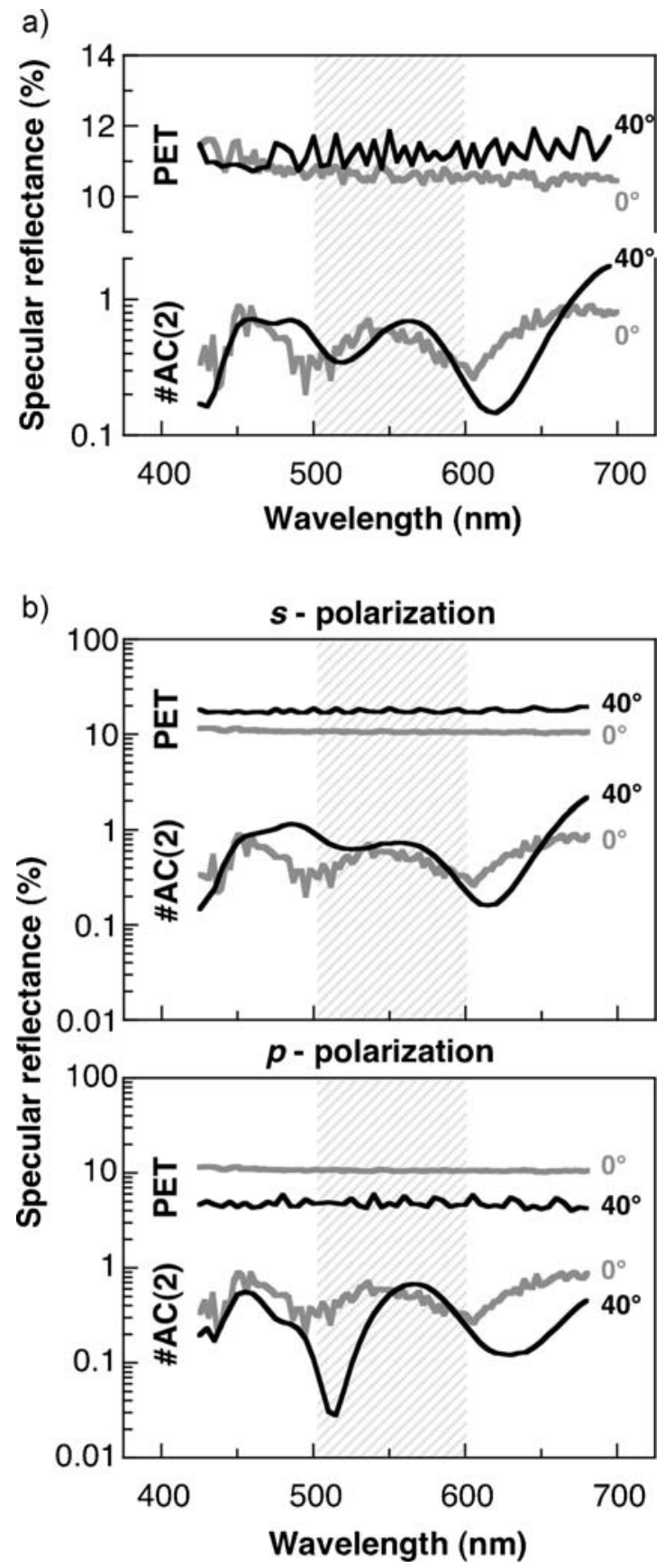

Figure 7. (a) Unpolarized and (b) polarized (with both $s$ - and $p$ polarized light) reflectance spectra measured at o and $40^{\circ}$ (gray and black lines, respectively) for a PET substrate coated with the $A C(2) A R$ coating. The corresponding spectra for the uncoated PET substrate are reported as reference.
Figure $7(b)]$. Note that for the $p$-state reflectance values as low as $0.02 \%$ are reached for a $40^{\circ}$ incidence.

\section{Conclusion}

In conclusion, we demonstrated that both high transparency and very low refractive index can be simultaneously achieved over the entire visible spectrum in hybrid polymer films coated with an industrial aqueous-based method on very large surfaces (from $\mathrm{cm}^{2}$ up to several $\mathrm{m}^{2}$ ). Using such nanoporous layers as basic building blocks, multilayer films with a total thickness of few $\mu \mathrm{m}$ and consisting of up to eight different layers can be fabricated in a single roll-to-roll process on flexible substrates. In particular, we designed and fabricated graded-index hybrid polymer coatings that, once deposited on a polymer substrate, successfully reduce its surface reflection from $10 \%$ to few $\%$, with theoretical reflectivity values that may reach some $0.01-0.001 \%$ depending on the multilayer structure. We highlight that, on one hand, after being coated on a flexible support, our films may be transferred onto other inorganic substrates (e.g., $\mathrm{Si} / \mathrm{SiO}_{2}$ wafers). On the other hand, both the film quality and its optical performances may be further improved by optimizing the coating process. Finally, our low- $n$ nanoporous layers can be combined in a complex multilayer stack with other polymer or nanoporous layers with $n$ values higher than 1.5 (e.g., our $\mathrm{TiO}_{2}$-based films), thus allowing the fabrication of graded-index films with more sophisticated index profiles ${ }^{[28]}$ and good AR properties for a large palette of both organic and inorganic substrates.

Acknowledgements: The authors thank M. Schär and M. Longchamp from the École Polytechnique Fédérale de Lausanne (EPFL, Switzerland), and M. Pauchard, G. Liechti, S. Ruegg, and J. Ketterer from Ilford Imaging Switzerland $\mathrm{GmbH}$ (Marly, Switzerland) for their help. This work was supported by the Swiss Confederation Innovation Promotion Agency (Commission pour la Technologie et l'Innovation - CTI) and the Swiss National Science Foundation (SNSF) through the CTI-8184.1 EPRP-IW and the SNSF200021-111856 projects, respectively.

Received: February 8, 2010; Revised: April 16, 2010; Published online: XXX; DOI: 10.1002/mame.201000045

Keywords: anti-reflection coatings; nanocomposites; nanoporous films; optics; water-soluble polymers

[1] S. Y. Yang, I. Ryu, H. Y. Kim, J. K. Kim, S. K. Jang, T. P. Russell, Adv. Mater. 2006, 18, 709.

[2] Z.-M. Oi, M. Wei, H. Matsuda, I. Honma, H. Zhou, Appl. Phys. Lett. 2007, 90, 011102. 
[3] S.-J. Ding, P.-F. Wang, X.-G. Wan, D. W. Zhang, J.-T. Wang, W. W. Lee, Mater. Sci. Eng., B 2001, 83, 130.

[4] M. S. Park, Y. Lee, J. K. Kim, Chem. Mater. 2005, 17, 3944.

[5] Photonic Crystals, K. Busch, S. Lölkes, R. B. Wehrspohn, H. Föll, Eds., Wiley-VCH, Weinheim 2004.

[6] K. Maex, M. R. Baklanov, D. Shamiryan, F. Iacopi, S. H. Brongersma, Z. S. Yanovitskaya, J. Appl. Phys. 2003, 93, 8793.

[7] R. D. Miller, Science 1999, 286, 421.

[8] D. Konjhodzic, S. Schröter, F. Marlow, Phys. Status Solidi A 2007, 204, 3676.

[9] H. Y. Peng, L. Ho, X. J. Yu, H. S. Kwok, J. Appl. Phys. 2004, 96, 1649.

[10] T. Tsutsui, M. Yahiro, H. Yokogawa, K. Kawano, M. Yokoyama, Adv. Mater. 2001, 13, 1149.

[11] EU 1492389-A1 (2004), Ilford Imaging Switzerland GmbH, invs.: R. Beer, R. Steiger, M. Schaer, L. Zuppiroli,

[12] Y.-H. Cheng, J.-L. Wu, C.-H. Cheng, K.-C. Syao, M.-C. M. Lee, Appl. Phys. Lett. 2007, 90, 091102.

[13] P. Oelhafen, A. Schüler, Sol. Energy 2005, 79, 110.

[14] A. G. Agrios, I. Cesar, P. Comte, M. K. Nazeeruddin, M. Grätzel, Chem. Mater. 2006, 18, 5395.

[15] A. Mihi, F. J. López-Alcaraz, H. Míguez, Appl. Phys. Lett. 2006, 88, 193110.

[16] S. Colodrero, A. Mihi, J. A. Anta, M. Ocaña, H. Míguez, J. Phys. Chem. C 2009, 113, 1150.

[17] E. Lancelle-Beltran, P. Prené, C. Boscher, P. Belleville, P. Buvat, C. Sanchez, Adv. Mater. 2006, 18, 2579.

[18] M. E. Calvo, S. Colodrero, T. C. Rojas, J. A. Anta, M. Ocaña, H. Míguez, Adv. Funct. Mater. 2008, 18, 2708.

[19] P. Kurt, D. Banerjee, R. E. Cohen, M. F. Rubner, J. Mater. Chem. 2009, 19, 8920.

[20] P. Pirasteh, J. Charrier, Y. Dumeige, P. Joubert, S. Haesaert, A. Chaillou, L. Haji, P. Le Rendu, T. P. Nguyen, Phys. Status Solidi A 2005, 202, 1712.

[21] P. Ferrand, R. Romestain, Appl. Phys. Lett. 2000, 77, 3535.

[22] J. S. Rayleigh, Proc. London Math. Soc. 1880 11, 51.

[23] J. Fraunhofer, "Versuche über die Ursachen des Anlaufens und Mattwerdens des Glases und die Mittel, denselben zuvorzukommen", in: J. von Fraunhofer's Gesammelte Schriften, Verlag der K. Akademie, Munich 1888.

[24] H. A. MacLeod, Thin-Film Optical Filters, Hilger, Bristol 1986.

[25] Polymer Data Handbook, J. E. Mark, Ed., Oxford University Press, New York 1999.

[26] Physics of Thin Films- Advances in Research and Development, Vol. 2, G. Hass, R. E. Thun, Eds., Academic Press, New York 1964

[27] R. K. Scott, J. B. Michael, Appl. Opt. 2003, 42, 4573.

[28] J.-O. Xi, M. F. Schubert, J. K. Kim, E. F. Schubert, M. Chen, S.-Y. Lin, W. Liu, J. A. Smart, Nat. Photonics 2007, 1, 176.

[29] Y.-F. Huang, S. Chattopadhyay, Y.-J. Jen, C.-Y. Peng, T.-A. Liu, Y.-K. Hsu, C.-L. Pan, H.-C. Lo, C.-H. Hsu, Y.-H. Chang, C.-S. Lee, K.H. Chen, L.-C. Chen, Nat. Nanotechnol. 2007, 2, 770.

[30] M.-J. Huang, C.-R. Yang, Y.-C. Chiou, R.-T. Lee, Sol. Energy Mater. Sol. Cells 2008, 92, 1352.
[31] I. Saarikoski, M. Suvanto, T. A. Pakkanen, Thin Solid Films 2008, 516, 8278.

[32] EU 2010308-A1 (2006), Commissariat Energie Atomique, invs.: B. Pintault, D. Guenadou, L. Bianchi, P. Belleville, K. Valle, C. Boscher, J. Toulch'oat

[33] P. Belleville, P. Prené, F. Mennechez, C. Bouigeon, Proceedings of the Society of Photo-Optical Instrumentation Engineers, Vol. 4804 , E. J. A. Pope, H. K. Schmidt, B. S. Dunn, S. Shibata, Eds., SPIE Conf. on Sol-Gel Optics VI, SPIE, Seattle 2002.

[34] C. J. Brinker, Y. Lu, A. Sellinger, H. Fan, Adv. Mater. 1999, 11 , 579.

[35] H. Hattori, Adv. Mater. 2001, 13, 51.

[36] X. T. Zhang, O. Sato, M. Taguchi, Y. Einaga, T. Murakami, A. Fujishima, Chem. Mater. 2005, 17, 696.

[37] F. Cebeci, Z. Wu, L. Zhai, R. E. Cohen, M. F. Rubner, Langmuir 2006, 22, 2856.

[38] S. E. Yancey, W. Zhong, J. R. Heflin, A. L. Ritter, J. Appl. Phys. 2006, 99, 034313.

[39] H. Jiang, K. Yu, Y. Wang, Opt. Lett. 2007, 32, 575.

[40] US 2006074172-A1 (2006), Optimax Tech Corp, Taiwan, invs.: A. J. Yang, R. Zhang

[41] US 2006099407-A1 (2006), Industrial Technology Research Institute, Taiwan, invs.: W.-J. Wang, Y.-P. Wang, Y.-C. Lee, J.-Y. Chen, H.-H. Shih

[42] S. Walheim, E. Schäffer, J. Mlynek, U. Steiner, Science 1999, 283, 520.

[43] M. Ibn-Elhaj, M. Schadt, Nature 2001, 410, 796.

[44] J. Hiller, J. D. Mendelsohn, M. F. Rubner, Nat. Mater. 2002, 1, 59.

[45] M. S. Park, Y. Lee, J. K. Kim, Chem. Mater. 2005, 17, 3944.

[46] M. Zhao, Z. Yang, D. Zhu, X. Jin, D. Huang, J. Opt. Soc. Am. B 2005, 22, 1330.

[47] K. Biswas, S. Gangopadhyay, H.-C. Kim, R. D. Miller, Thin Solid Films 2006, 514, 350.

[48] W. Joo, M. S. Park, J. K. Kim, Langmuir 2006, 22, 7960.

[49] J. Cho, J. Hong, K. Char, F. Caruso, J. Am. Chem. Soc. 2006, 128, 9935.

[50] S. Kim, J. Cho, K. Char, Langmuir 2007, 23, 6737.

[51] EU 1655348-A1 (2006), Ilford Imaging Switzerland GmbH, invs.: U. Fuerholz, V. Ruffieux, M. Schaer

[52] See http://www.ilford.com/.

[53] A. Röseler, Infrared Spectroscopic Ellipsometry, AkademieVerlag, Berlin 1990.

[54] M. Born, E. Wolf, Principles of Optics, Cambridge University Press, Cambridge 1998.

[55] D. E. Aspnes, J. B. Theeten, F. Hottier, Phys. Rev. B 1979, 20, 3292.

[56] A. Sihvola, Electromagnetic Mixing Formulas and Applications, The Institution of Electrical Engineers, London 1999.

[57] C. K. Charalambos, I. S. Dimitrios, Appl. Opt. 2002, 41, 3978.

[58] P. Beckmann, A. Spizzichino, The Scattering of Electromagnetic Waves from Rough Surfaces, Pergamon Press, Oxford 1963. 